\title{
ICI Cancellation in OFDM Systems by Frequency Offset Reduction
}

\author{
Naveed Ur Rehman Zhang Lei Muhammad Zahid Hammad \\ Department of Information and Communication Harbin Engineering University Harbin, China \\ Corresponding author:p07010@nu.edu.pk
}

\begin{abstract}
With the rapid growth of digital communication in recent years, the need for high speed data transmission is increased. Moreover, future wireless systems are expected to support a wide range of services which includes video, data and voice. OFDM is a promising candidate for achieving high data rates in mobile environment because of its multicarrier modulation technique and ability to convert a frequency selective fading channel into several nearly flat fading channels. Now OFDM is being widely used in wireless communications standards, such as IEEE 802.11a, the multimedia mobile access communication (MMAC), and the HIPERLAN/2. However, one of the main disadvantages of OFDM is its sensitivity against carrier frequency offset which causes inter carrier interference (ICI). A well-known problem of orthogonal frequency division multiplexing (OFDM), however, is its sensitivity to frequency offset between the transmitted and received signals, which may be caused by Doppler shift in the channel, or by the difference between the transmitter and receiver local oscillator frequencies. This carrier frequency offset causes loss of orthogonality between sub-carriers and the signals transmitted on each carrier are not independent of each other. The orthogonality of the carriers is no longer maintained, which results in inter-carrier interference (ICI). The undesired ICI degrades the performance of the system. Depending on the Doppler spread in the channel and the block length chosen for transmission, ICI can potentially cause a severe deterioration of quality of service (QOS) in OFDM systems. ICI mitigation techniques are essential in improving the performance of an OFDM system in an environment which induces frequency offset error in the transmitted signal. The comparisons of these schemes in terms of various parameters will be useful in determining the choice of ICI mitigation techniques for different applications and mobile environments. This project investigates an efficient ICI cancellation method termed ICI self-cancellation scheme for combating the impact of ICI on OFDM systems. The ICI self-cancellation scheme is a technique in which redundant data is transmitted onto adjacent sub-carriers such that the ICI between adjacent sub-carriers cancels out at the receiver. The main idea is one data symbol is modulated onto a group of adjacent subcarriers with a group of weighting coefficients. By doing so, the ICI signals generated within a group can be self-cancelled each other. At the receiver side, by linearly combining the received signals on these subcarriers with proposed coefficients, the residual ICI contained in the received signals can then be further reduced. Although the proposed scheme causes a reduction in bandwidth efficiency, it can be compensated, by using larger signal alphabet sizes in modulation. The average carrier-to-interference power ratio (CIR) is used as the ICI level indicator, and a theoretical CIR expression is derived for the proposed scheme. The proposed scheme provides significant CIR improvement, which has been studied theoretically and supported by simulations. Simulation results show that under the condition of the same bandwidth efficiency and larger frequency offsets, the proposed OFDM system using the ICI self-cancellation scheme per- forms much better than standard OFDM systems in AWGN channel with large Doppler frequencies. In addition, since no channel equalization is needed for reducing ICI, the proposed scheme is therefore beneficial in implementation issue without increasing system complexity
\end{abstract}

\section{INTRODUCTION}

The increasing demand for very high data rate wireless data transmission calls for technologies which make use of the available electromagnetic spectrum in the most intelligent way. Key objectives are spectrum efficiency (bits per second per Hertz), robustness against multipath propagation, range, power consumption, and implementation complexity. These objectives are often conflicting, so techniques and implementations are sought which offer the best possible tradeoff between them. The Internet revolution has created the need for wireless technologies that can deliver data at high speeds in a spectrally efficient manner. However, supporting such high data rates with sufficient robustness to radio channel impairments requires careful selection of modulation techniques. Currently, the most suitable choice appears to be OFDM (Orthogonal Frequency Division Multiplexing).Orthogonal frequency division multiplexing (OFDM) is becoming the chosen modulation technique for wire- less communications. OFDM can provide large data rates with sufficient robustness to radio channel impairments.

Orthogonal Frequency Division Multiplexing OFDM is a special form of multicarrier modulation technique which is used to generate waveforms that are mutually orthogonal. In an OFDM scheme, a large number of orthogonal, overlapping, narrow band sub-carriers are transmitted in parallel. These carriers divide the available transmission bandwidth. The separation of the sub- carriers is such that there is a very compact spectral 
utilization. With OFDM, it is possible to have overlapping sub channels in the frequency domain, thus increasing the transmission rate. In order to avoid a large number of modulators and filters at the transmitter and complementary filters and demodulators at the receiver, it is desirable to be able to use modern digital signal processing techniques, such as fast Fourier transform (FFT). After more than forty years of research and development carried out in different places, OFDM is now being widely implemented in high-speed digital communications. OFDM has been accepted as standard in several wire line and wireless applications. Due to the recent advancements in digital signal processing (DSP) and very large- scale integrated circuits (VLSI) technologies, the initial obstacles of OFDM implementations do not exist anymore. In a basic communication system, the data are modulated onto a single carrier frequency. The available bandwidth is then totally occupied by each symbol. This kind of system can lead to inter-symbol-interference (ISI) in case of frequency selective channel. The basic idea of OFDM is to divide the available spectrum into several orthogonal sub channels so that each narrowband sub channels experiences almost flat fading. Many research centers in the world have specialized teams working in the optimization of OFDM systems. The attraction of OFDM is mainly because of its way of handling the multipath interference at the receiver. Multipath phenomenon generates two effects (a) Frequency selective fading and

(b) Intersymbol interference (ISI). The flatness" perceived by a narrowband channel overcomes the frequency selective fading. On the other hand, modulating symbols at a very low rate makes the symbols much longer than channel impulse response and hence reduces the ISI. Use of suitable error correcting codes provides more robustness against frequency selective fading. The insertion of an extra guard interval between consecutive OFDM symbols can reduce the effects of ISI even more. The use of FFT technique to implement modulation and demodulation functions makes it computationally more efficient. OFDM systems have gained an increased interest during the last years. It is used in the European digital broadcast radio system, as well as in wired environment such as asymmetric digital subscriber lines (ADSL). This technique is used in digital subscriber lines (DSL) to provide high bit rate over a twisted-pair of wires.

The major advantages of OFDM are its ability to convert a frequency selective fading channel into several nearly flat fading channels and high spectral efficiency. However, one of the main disadvantages of OFDM is its sensitivity against carrier frequency offset which causes attenuation and rotation of sub- carriers, and inter carrier interference (ICI) [1,2]. The undesired ICI degrades the performance of the system.

\section{MOTIVATION}

OFDM is robust in adverse channel conditions and allows a high level of spectral efficiency. Multiple access techniques which are quite developed for the single carrier modulations (e.g. TDMA, FDMA) had made possible of sharing one communication medium by multiple number of users simultaneously. The sharing is required to achieve high capacity by simultaneously allocating the available bandwidth to multiple users without severe degradation in the performance of the system. FDMA and TDMA are the well-known multiplexing techniques used in wireless communication systems.

While working with the wireless systems using these techniques, various problems encountered are:

1. Multi-path fading

2. Time dispersion which lead ISI

3. Lower bit rate capacity

4. Requirement of larger transmit power for high bit rate

5. Less spectral efficiency. Disadvantage of FDMA technique is its Bad Spectrum Usage. Disadvantages of TDMA technique is Multipath Delay spread problem. In a typical terrestrial broadcasting, the

Transmitted signal arrives at the receiver using various paths of different lengths. Since multiple versions of the signal interfere with each other, it becomes difficult to extract the original information.

Orthogonal Frequency Division Multiplexing (OFDM) has recently gained fair degree of prominence among modulation schemes due to its intrinsic robust- ness to frequency selective Multipath fading channels. OFDM system also provides higher spectrum efficiency and supports high data rate transmission. This is one of the main reasons to select OFDM a candidate for systems such as Digital Audio Broadcasting (DAB), Digital Video Broadcasting (DVB), Digital Subscriber Lines (DSL), and Wireless local area networks (HiperLAN/2), and in IEEE 802.11a, IEEE 802.11g.

The focus of future fourth-generation $(4 \mathrm{G})$ mobile systems is on supporting high data rate services such as deployment of multi-media applications which involve voice, data, pictures, and video over the wireless networks. At this moment, the data rate envisioned for $4 \mathrm{G}$ networks is $1 \mathrm{~GB} / \mathrm{s}$ for indoor and $100 \mathrm{Mb} / \mathrm{s}$ for outdoor environments. Orthogonal frequency division multiplexing (OFDM) is a promising candidate for $4 \mathrm{G}$ systems because of its robustness to the multipath environment.

The focus of future fourth-generation $(4 \mathrm{G})$ mobile systems is on supporting high data rate services such as deployment of multi-media applications which involve voice, data, pictures, and video over the wireless 
networks. At this moment, the data rate envisioned for $4 \mathrm{G}$ networks is $1 \mathrm{~GB} / \mathrm{s}$ for indoor and $100 \mathrm{Mb} / \mathrm{s}$ for outdoor environments. Orthogonal frequency division multiplexing (OFDM) is a promising candidate for $4 \mathrm{G}$ systems because of its robustness to the multipath environment.

\section{LITERATURE SURVEY}

In 1971, Weinstein and Ebert proposed a modified OFDM system [7] in which the discrete Fourier Transform (DFT) was applied to generate the orthogonal subcarriers waveforms instead of the banks of sinusoidal generators. Their scheme reduced the implementation complexity significantly, by making use of the inverse DFT (IDFT) modules and the digital- to-analog converters. In their proposed model, baseband signals were modulated by the IDFT in the transmitter and then demodulated by DFT in the receiver. Therefore, all the subcarriers were overlapped with others in the frequency domain, while the DFT modulation still assures their orthogonally. $[7,18]$

Cyclic prefix (CP) or cyclic extension was first introduced by Peled and Ruiz in 1980 [8] for OFDM systems. In their scheme, conventional null guard interval is substituted by cyclic extension for fully-loaded OFDM modulation. As a result, the orthogonally among the subcarriers was guaranteed. With the trade-off of the transmitting energy efficiency, this new scheme can result in a phenomenal ISI (Inter Symbol Interference) reduction. Hence it has been adopted by the current IEEE standards. In 1980, Hirosaki introduced an equalization algorithm to suppress both inter symbol interference (ISI) and ICI [9], which may have resulted from a channel distortion, synchronization error, or phase error. In the meantime, Hirosaki also applied QAM modulation, pilot tone, and trellis coding techniques in his high- speed OFDM system, which operated in voiceband spectrum.

In 1985, Cimini introduced a pilot-based method to reduce the interference emanating from the multipath and co-channels [10]. In the 1990s, OFDM systems have been exploited for high data rate communications. In the IEEE

802.11 standard, the carrier frequency can go up as high as $2.4 \mathrm{GHz}$ or $5 \mathrm{GHz}$. Researchers tend to pursue OFDM operating at even much higher frequencies nowadays. For example, the IEEE 802.16 standard proposes yet higher carrier frequencies ranging from $10 \mathrm{GHz}$ to $60 \mathrm{GHz}$. However, one of the main disadvantages of OFDM is its sensitivity against carrier frequency offset which causes intercarrier interference (ICI). The undesired ICI degrades the perfor- mance of the system. ICI self- cancellation is a scheme that was introduced by Yuping Zhao and Sven-Gustav Hggman in 2001 to combat and suppress ICI in OFDM.

\section{CONTRIBUTION}

Using MATLAB, simulation of OFDM was done with different modulation techniques using self-cancellation technique. The digital modulation schemes such as BPSK and QPSK were selected to assess the performance of the de- signed OFDM system by finding their bit error rate (BER) for different values of signal to noise ratio (SNR). In this project, I have focused on the problem of ICI reduction using self-cancellation scheme and compared with standard OFDM system. Different modulation techniques are considered for ICI reduction and compared with each other for their performances. I have also briefly discussed OFDM and its advantages and disadvantages as compared to single carrier modulation technique.

\section{METHODS OF ICI REDUCTION}

1. Frequency domain equalization

2. Time domain windowing

3. Pulse shaping

4. ICI self cancellation

\section{Frequency Domain Equalization}

The fading distortion in the channel causes ICI in the OFDM demodulator. The pattern of ICI varies from frame to frame for the demodulated data but remains invariant for all symbols within a demodulated data frame.// Compensation for fading distortion in the time domain introduces the problem of noise enhancement. So frequency domain equalization process is approached for reduction of ICI by using suitable equalization techniques. We can estimate the ICI for each frame by inserting frequency domain pilot symbols in each frame as shown in figure 3.6. The equalizer co-efficient for eliminating ICI in 


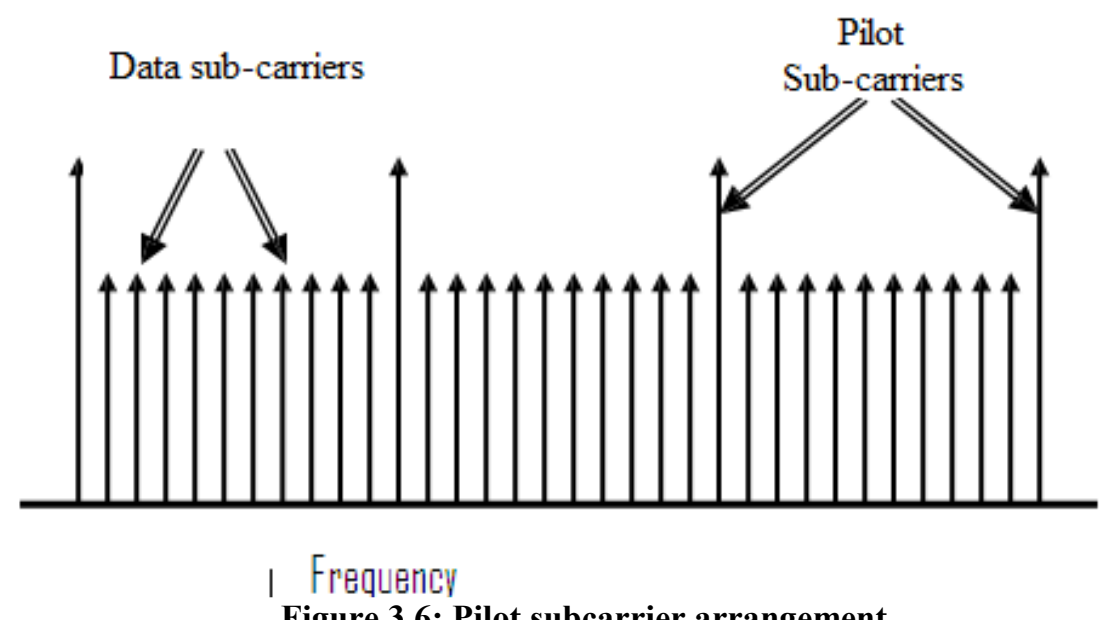

Figure 3.6: Pilot subcarrier arrangement.

The frequency domain can be derived from the pattern of the pilot symbol $\&$ hence a suitable equalizer can be constructed.

\section{Drawbacks}

It can only reduce the ICI caused by fading distortion which is not the major source of ICI. The major source of ICI is due to the frequency mismatch be- tween the transmitter and receiver, and the Doppler shift. The above method cannot address to it.

Again it is only suitable for flat fading channels, but in mobile communication the channels are frequency selective fading in nature because of multipath components. Here also the channel needs to be estimated for every frame. Estimation of channel is complex, expensive \& time consuming. Hence the method is not effective one.

\section{Time Domain Windowing}

We know that OFDM signal has widely spread power spectrum. So if this signal is transmitted in a band limited channel, certain portion of the signal spectrum will be cut off, which will lead to inter carrier interference. To

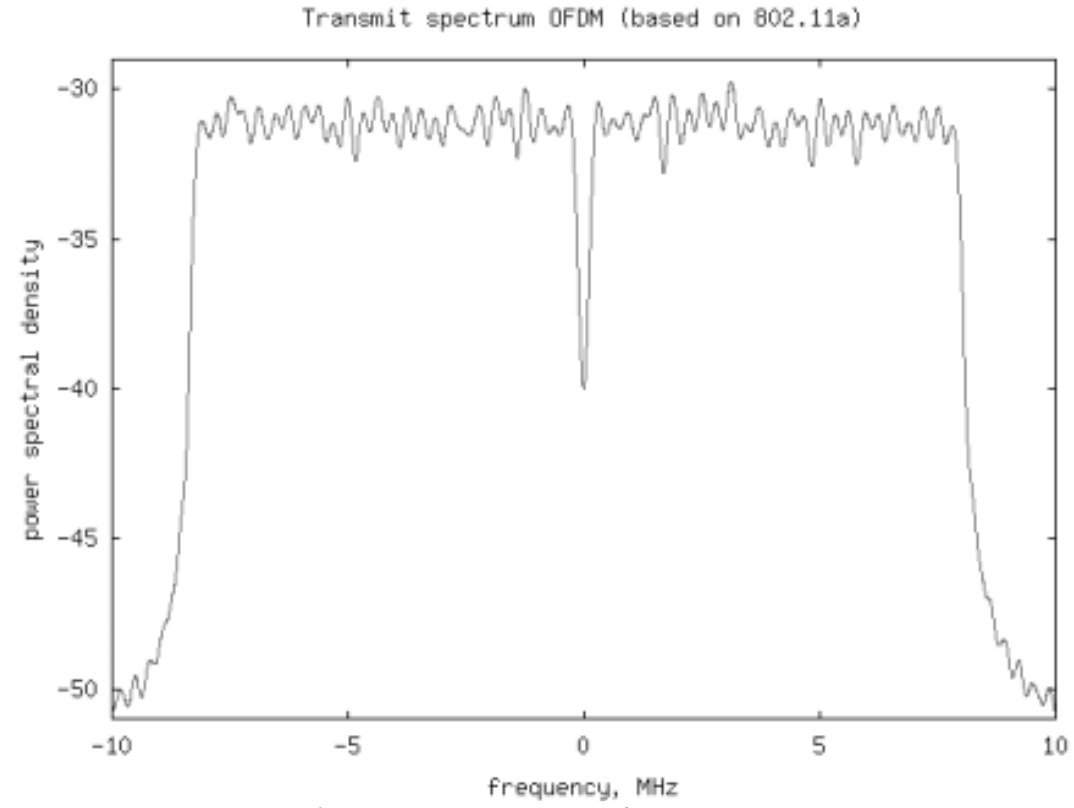

Figure 3.7: Spectrum of 64-QAm.

Diminish the interference the spectrum of the signal wave form need to be more concentrated. This is achieved by windowing the signal. Basically windowing is the process of multiplying a suitable function to the transmitted signal wave form. The same window is used in the receiver side to get back the original signal. The 
IC1 will be eliminated if the product of the window functions satisfies the Nyquists vestigial symmetry criterion. The IC1 will be eliminated if the product of the window functions satisfies the Nyquists vestigial symmetry criterion.

\section{Frame by frame windowing}

The conventional frame-by-frame time limited orthogonal multicarrier signal $\mathrm{S}(\mathrm{t})$ can be expressed as:

$s(t)=\infty \quad N-1$

$\ldots w(t-k T) \alpha n, k \operatorname{ejnw} \delta(t-k T)$

$k=-\infty n=0$

where $\alpha n, k$ is a complex sequence,$w \delta n=(0,1,2, \cdots, N-1)$ are carrier frequencies and they are equally spaced with $w \delta$ The window function $\mathrm{w}(\mathrm{t})$ has a length of $\mathrm{T}$ and it modifies the waveform of the multicarrier signal in each frame.

The IC1 for this multicarrier signal can be avoided if the window function $\mathrm{w}(\mathrm{t})$

and the carrier separation are correctly chosen. In order to provide a matched receiver for the transmitted signal, the window functions in both the trans- mitter and the receiver are selected to be equal. The IC1 can be determined by examining the cross correlation between two carriers of the transmitted signals. For a complex sequence $\alpha n . k$ the condition for eliminating the IC1 is given by:

$$
\begin{array}{ll}
\alpha_{n, k} \alpha_{m, k} & , T w^{2} e^{-j w \delta(n-m)} d t=0 \quad \text { form } f=n \cdots \\
& \\
& -T \\
& \\
&
\end{array}
$$

and if the function $w 2(t)$ meets the Nyquists vestigial symmetry criterion, this condition will be satisfied. If $\mathrm{T}$ is a time parameter and $\mathrm{T} / 2 \leq \mathrm{T} \leq \mathrm{T}$.

Then the window function $\mathrm{w}(\mathrm{t})$ which satisfies the Nyquist criterion will be described by

$$
w^{2}(t)=x(t)+Y(t) \quad T / 2 \leq T \leq T / 2
$$

otherwise

Here $\mathrm{x}(\mathrm{t})$ is a rectangular window function over [-T/2, T/2] and $\mathrm{y}(\mathrm{t})$ is an even function with odd symmetry about $\mathrm{T} / 2$ and $\mathrm{T} / 2$. It is known that the raised cosine function satisfies the Nyquist criterion, and so this function can be considered as $\mathrm{y}(\mathrm{t})$. Here $\mathrm{T}$ is defined as $\mathrm{T}=\mathrm{T}(1+\alpha)$ and $\alpha$ is the roll-off parameter of the raised cosine function. The IC1 free condition requires the frequency separation between adjacent carriers to be :

$$
w_{\delta}=\frac{2 \pi(1+\alpha)}{T}
$$

So, the total required bandwidth for $\mathrm{N}$ carriers is about $(1+\alpha) / T S$ where $T S$ is the symbol interval of the input sequence and $\mathrm{T}=\mathrm{NTS}$

\section{Drawbacks}

It can only reduce the ICI caused by band limited channel which is not the major source of ICI. The major source of ICI is due to the frequency mis- match between the transmitter and receiver, and the Doppler shift. The above method cannot address to it. Windowing is done frame by frame \& hence it reduces the spectral efficiency to a large extent. Hence the method is not effective one.

\section{Pulse Shaping}

As we have seen in the OFDM spectrum that each carrier consist of a main lobe followed by a number of side lobes with reducing amplitude. As long as orthogonality is maintained there is no interference among the carriers because at the peak of the every carrier, there exist a spectral null. That is at that point the component of all other carriers is zero. Hence the individual carrier is easily separated. When there is a frequency offset the orthogonality is lost because now the spectral null does not coincide to the peak of the individual carriers.

carriers which is called ICI power. The ICI power will go on increasing as the frequency offset increases. The purpose of pulse shaping is to reduce the side lobes. If we can reduce the side lobe significantly then the ICI power will also be reduced significantly. 


\section{Drawback}

Complex in implementation

\section{ICI Self Cancellation}

It is seen that the difference between the ICI co-efficient of two consecutive sub- carriers are very small. This makes the basis of ICI self-cancellation. Here one data symbol is not modulated in to one sub-carrier, rather at least in to two consecutive sub-carriers. If the data symbol a is modulated in to the 1st sub-carrier then -a is modulated in to the 2nd sub- carrier. Hence the ICI generated between the two sub-carriers almost mutually cancels each other. This method is suitable for multipath fading channels as here no channel estimation is required. Because in multipath case channel estimation fails as the channel changes randomly.

\section{ICI Cancelling Modulation}

In an OFDM communication system, assuming the channel frequency offset normalized by the subcarrier separation is, and then the received signal on subcarrier $\mathrm{k}$ can be written as:

$$
N-1
$$

$$
Y(k)=X(k) S(0)+\quad-\quad X(l) S(l-k)+n_{k}, \quad k=0,1, \cdots N-1
$$

$$
\begin{gathered}
l=0, l f \\
=k
\end{gathered}
$$

Where $\mathrm{N}$ is the total number of the subcarriers, $\mathrm{X}(\mathrm{k})$ denotes the transmitted symbol for the kth subcarrier and $\mathrm{nk}$ is an additive noise sample. The first term in the right-hand side of (3.15) represents the desired signal. The second term is the ICI components. The sequence $\mathrm{S}(1-\mathrm{k})$ is defined as the ICI coefficient between lth and kth subcarriers, which can be expressed as:

$$
\mathbf{S}(\mathbf{l}-\mathbf{k})=\begin{array}{ll}
\frac{\sin (\pi(\mathbf{l}+s-\mathbf{k}))}{\mathbf{N} \sin \left(\frac{\pi}{\mathbf{N}}(s-\mathbf{k})\right)} & \begin{array}{l}
\mathbf{e}^{\mathbf{j} \pi(\mathbf{1}-\underline{\mathbf{1}}} \\
(\mathbf{l}+s+\mathbf{k}))
\end{array} \\
\ldots & \mathbf{N}
\end{array}
$$

It is seen that the difference of ICI coefficient between two consecutive sub- carrier $(S(l-k)$ and $S(l+1-k)$ is very small. Therefore, if a data pair $(\mathrm{a},-\mathrm{a})$ is modulated onto two adjacent subcarriers $(1,1+1)$, where a is a complex data, then the ICI signals generated by the subcarrier 1 will be cancelled out significantly by the ICI generated by subcarrier $1+1$.[14]

Assuming the transmitted symbols are such that $\mathrm{X}(1)=-\mathrm{X}(0), \mathrm{X}(3)=-\mathrm{X}(2), \cdots, \mathrm{X}(\mathrm{N}-1)=-\mathrm{X}(\mathrm{N}-2)$, then the received signal on subcarrier $\mathrm{k}$ becomes

$$
\begin{gathered}
Y^{t}(k)=\underbrace{N-2}_{\substack{l=0, l=e v \\
e n}} X(l)\left[S(l-k)-S(l+1-k)+n_{k}\right. \\
\quad
\end{gathered}
$$

Similarly the received signal on subcarrier $\mathrm{k}+1$ becomes

$$
N-2
$$

$$
Y^{t}(k+1)=\quad-\quad X(l)\left[S(l-k-1)-S(l-k)+n_{k+1}\right.
$$

$$
l=0, l=e v
$$

en

In such a case, the ICI coefficient is denoted as

$$
S t(l-k)=S(l-k)-S(l+1-k)
$$

It is found that $S t(l+k)<<S(l-k)$, which is shown in figure 3.8 


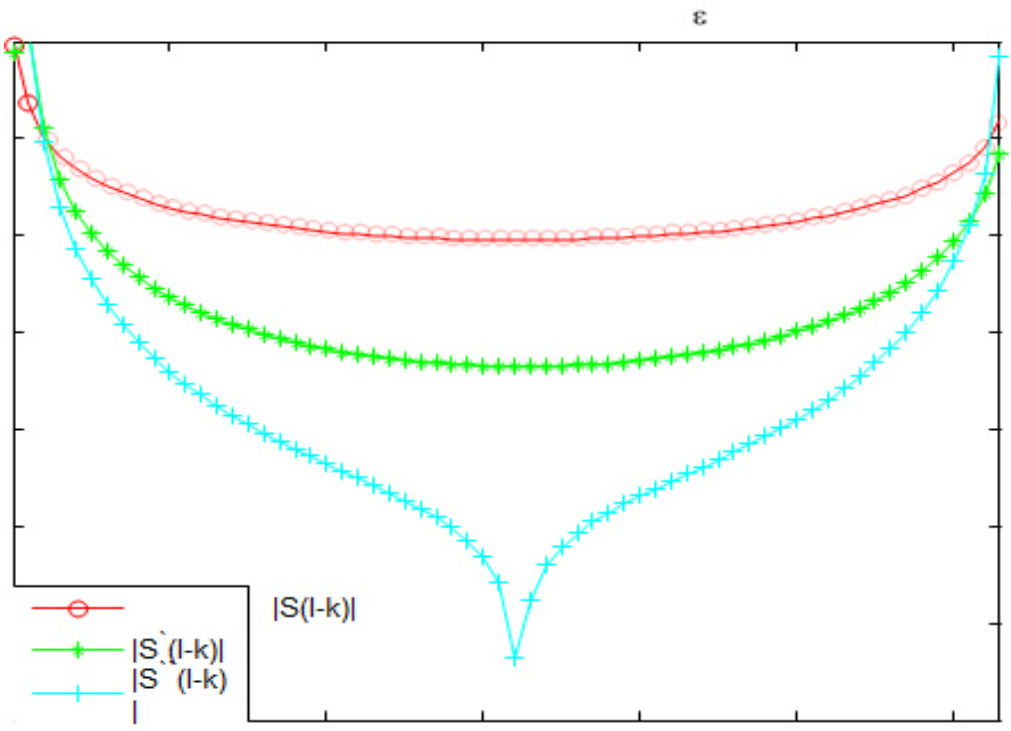

Figure 3.8: Comparison between $[S(l-k) \operatorname{Sr}(l-k)$ and $\operatorname{Srr}(l-k)]$.

\section{ICI Cancelling Demodulation}

To further reduce ICI, ICI cancelling demodulation is done. The demodulation is suggested to work in such a way that each signal at the $(k+1)$ th subcar- rier (now $\mathrm{k}$ denotes even number) is multiplied by -1 and then summed with the one at the kth subcarrier. Then the resultant data sequence is used for making symbol decision. It can be represented as $Y t t(k+1)=Y t(k)-Y t(k+1)$

The corresponding ICI coefficient then becomes

$$
Y t t(k+1)=X(l)[-S(l-k-1)+2 S(l-k)-S(l-k+1)]+n k+n k+1(3.20)
$$

$$
S t t(l-k)=-S(l-k-1)+2 S(l-k)-S(l-k+1)
$$

Figure 3.8 shows the amplitude comparison of $S(l-k)$, St $(l-k)$ and $\operatorname{Stt}(l-k)$ for $\mathrm{N}=64$ and $s=0.3$ for the majority of $(l-k)$ values, $\operatorname{Stt}(l-k)$ is much

Smaller than $S(l-k)$, and the $\operatorname{Stt}(l-k)$ is even smaller than $S t(l-k)$ Thus, the ICI signals become smaller when applying ICI cancelling modulation. On the other hand, the ICI canceling demodulation can further reduce the residual ICI in the received signals.[14] The combined ICI cancelling modulation and demodulation method is called the ICI self- cancellation scheme.

\section{Drawback}

The major drawback of this method is the reduction in band width efficiency as same symbol occupies two subcarriers.

\section{Merits of this technique}

1. It is suitable for multipath fading channels

2. It is also suitable for flat channels

3. Channel estimation is not required

4. Channel equalization is not required

5. It is simple in implementation

6. It is less complex and effective

\section{Conclusion}

In this project, we have analyzed the OFDM system in the presence of different frequency offset between the transmitter and the receiver in terms of the Carrier-to- Interference ratio (CIR) and the bit error rate (BER) performance. Inter-carrier interference (ICI) which occurs because of frequency offset degrades the performance of the OFDM system. Different methods have been explored in this project for the cancellation of the ICI. After lot of research ICI self-cancellation (SC) has been proposed as the most efficient and optimized technique to cope with ICI.

The extended Kalman filter (EKF) method and some other techniques for estimation and cancellation of the frequency offset have been investigated in this project. The choice of which method to employ depends on the specific application. For example, self-cancellation does not require very complex hardware or software for 
implementation. However, it is not bandwidth efficient as there is a redundancy of 2 for each carrier. The ML method also introduces the same level of redundancy but provides better BER performance, since it accurately estimates the frequency offset. Its implementation is more complex than the SC method. On the other hand, the EKF method does not reduce bandwidth efficiency as the frequency offset can be estimated from the preamble of the data sequence in each OFDM frame. However, it has the most complex implementation of the two methods. In addition, this method requires a training sequence to be sent before the data symbols for estimation of the frequency offset. It can be adopted for the receiver design for IEEE 802.11a because this standard specifies preambles for every OFDM frame. The preambles are used as the training sequence for estimation of the frequency offset. In this project, the simulations were performed in an AWGN channel. This model can be easily adapted to a flat-fading channel with perfect channel estimation. Further work can be done by performing simulations to investigate the performance of these ICI cancellation schemes in multipath fading channels without perfect channel information at the receiver. In this case, the multipath fading may hamper the performance of these ICI cancellation schemes.

\section{Scope OF Future Work}

Following are the areas of future study which can be considered for further research work.

1. In this work the BER performance of the OFDM system is evaluated considering different versions of QAM Modulation technique. It can be tested with other modulation systems such as BPSK QPSK and GMSK. ICI reduction using self cancellation technique can be used for COFDM (Coded OFDM) Systems.? ]

2. This self cancellation technique can also be applied under different chan- nel conditions such as Rayleigh fading channel, urban area channel, rural area channel etc.

3. This self cancellation scheme can be extended to MIMO-OFDM systems and a Bayesian Statespace approach (SIS) to combat inter-carrier inter- ference in OFDM systems can be used.

4. The sequential Monte Carlo (SMC) method called sequential importance sampling.

(SIS) can be implemented which requires very lower computational complex- ity and estimates accurately high value frequency offsets. However, the SIS performs slightly better, which is expected due to the nonlinearity of the states- pace and it is bandwidth efficiency scheme.[2]

\section{REFERENCES}

[1] Charan Langton, Intuitive Guide to Principles of communications, 2004.

[2] Anis Salwa Osman(2006), Ber performance study of orthogonal frequency division Multiplexing(OFDM).

[3] History of OFDM.

[4] http://www.smsmatrix.com

[5] http://www.radio-electronics.com

[6] Srabani Mohapatra and Susmita Das(2009),Performance Enhancement of OFDM System with ICI Reduction Technique.

[7] Brahmaji T.A.R.K(2009),An efficient ICI cancellation Technique For OFDM Communication Systems.

[8] Ravitej Amasa(2009), Inter Carrier Interference Cancellation In OFDM Systems.

[9] S.Driz and Bouzani ,Inter-Carrier Interference Cancellation for OFDM systems.

[10] B.Satish Kumar and K.R. Shankar Kumar and R.Radhakirshna(2009),An Efficient Inter-carrier Interference Cancellation Schemes for OFDM Sys- tems.

[11] http://i.cmpnet.com/digitaltvdesignline/2006/2007/05/MFig7.jpg .

[12] Lou I.ILUNGA,Adaptive ,Turbo Coded OFDM.

[13] Eduardo Heras Misguel(October,2010),Fiber Based Orthogonal Frequency Division Multiplexin Transmission System .

[14] R.Kumar and Dr.S.Malarvizhi,Reduction Of Intercarrier Interference in OFDM Systems.

[15] Ramjee Parsad(2004),OFDM for Wireless Communication Systems.

[16] Mohammad Zavid Parvez and Md.Abdullah AL Baki,Peak to Average Power Ratio(PARR) Reduction in OFDM Based Radio Systems.

[17] Wan Fariza Binti Paiza,(April 2006) ,BER Performance of PSK-Based Digital Modulation Schemes in Multipath Fading Envirnoment. 
The IISTE is a pioneer in the Open-Access hosting service and academic event management. The aim of the firm is Accelerating Global Knowledge Sharing.

More information about the firm can be found on the homepage:

http://www.iiste.org

\section{CALL FOR JOURNAL PAPERS}

There are more than 30 peer-reviewed academic journals hosted under the hosting platform.

Prospective authors of journals can find the submission instruction on the following page: http://www.iiste.org/journals/ All the journals articles are available online to the readers all over the world without financial, legal, or technical barriers other than those inseparable from gaining access to the internet itself. Paper version of the journals is also available upon request of readers and authors.

\section{MORE RESOURCES}

Book publication information: http://www.iiste.org/book/

\section{IISTE Knowledge Sharing Partners}

EBSCO, Index Copernicus, Ulrich's Periodicals Directory, JournalTOCS, PKP Open Archives Harvester, Bielefeld Academic Search Engine, Elektronische Zeitschriftenbibliothek EZB, Open J-Gate, OCLC WorldCat, Universe Digtial Library, NewJour, Google Scholar

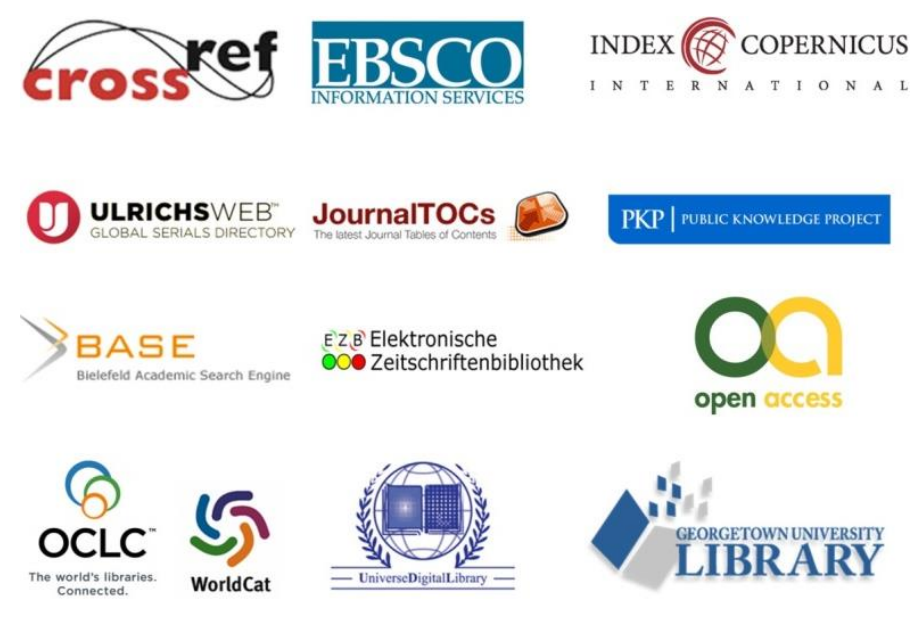

